\title{
Transmittivity of a Bose-Einstein condensate on a lattice: interference from period doubling and the effect of disorder
}

\author{
P. Vignolo ${ }^{1}$, Z. Akdeniz ${ }^{1,2}$ and M.P. Tosi ${ }^{1}$ \\ 1 NEST-INFM and Classe di Scienze, Scuola Normale Superiore, \\ I-56126 Pisa, Italy \\ ${ }^{2}$ Departement of Physics, University of Istanbul, Istanbul, Turkey.
}

\begin{abstract}
We evaluate the particle current flowing in steady state through a Bose-Einstein condensate subject to a constant force in a quasi-onedimensional lattice and to attractive interactions from fermionic atoms that are localized in various configurations inside the lattice wells. The system is treated within a BoseHubbard tight binding model by an out-of-equilibrium Green's function approach. A new band gap opens up when the lattice period is doubled by locating the fermions in alternate wells and yields an interference pattern in the transmittivity on varying the intensity of the driving force. The positions of the transmittivity minima are determined by matching the period of Bloch oscillations and the time for tunnelling across the band gap. Massive disorder in the distribution of the fermions will wash out the interference pattern, but the same period doubling of the lattice can be experimentally realized in a four-beam set-up. We report illustrative numerical results for a mixture of ${ }^{87} \mathrm{Rb}$ and ${ }^{40} \mathrm{~K}$ atoms in an optical lattice created by laser beams with a wavelength of $763 \mathrm{~nm}$.
\end{abstract}

PACS numbers: 05.30.-d, 03.75.Lm 


\section{Introduction}

A quasi-onedimensional (1D) optical lattice created by the interference of two counterpropagating laser beams and superposed on a highly elongated magnetic trap provides an almost ideal periodic potential for an atomic gas. A mixture of a BoseEinstein condensate and a degenerate fermion gas is an interesting system to study in such a confinement. The interactions can result in the spatial phase separation of the two components [1] or in the collapse of the fermionic component [2] and generally induce strong changes in the atomic dynamics 3 , depending on the values of the boson-boson and boson-fermion coupling constants.

A number of experiments have examined properties of ultracold bosonic atoms and condensates in optical lattices: Bloch oscillations [4] [5], Landau-Zener tunnelling [6], squeezed states [7, Josephson-like oscillations [8, a superfluid-toMott insulator transition [9], and 1D band structure [10] have all been observed. Theoretically, these effects have been studied by a variety of approaches such as the Gross-Pitaevski equation [11, the discretised non-linear Schrödinger equation [12], the Bogoliubov-de Gennes equations [13], the direct diagonalization of the Bose-Hubbard Hamiltonian [14, and quantum MonteCarlo simulations [15.

In this work we study the transport properties of the bosons in a boson-fermion mixture at zero temperature under the action of a constant driving force inside a 1D lattice. A constant acceleration can be imparted to the boson condensate by using a frequency-chirped optical lattice [17. For a suitable choice of the system parameters the tunnelling of the fermionic atoms across the lattice can be neglected and their presence simply modifies the well depths seen by the bosons. The interesting cases are those in which the fermionic component introduces additional periodicities or generates disorder in the lattice potential. All these modifications in the lattice potential could be more easily realized in the laboratory by using extra laser beams and/or speckle [16. However, we choose to focus on more sofisticated experimental situations where the additional periodicity or the disorder is induced by the fermions, with the aim to study the magnitude of these effects in such a system and to address our numerical results to experimental groups who investigate quantum transport in boson-fermion mixtures.

We describe the system by means of a 1D Bose-Hubbard Hamiltonian, using a renormalization of the scattering lengths to embody the transverse confinement [18. The Hamiltonian is not explicitly solved, but the whole lattice is reduced to a single dimer by a renormalization/decimation procedure 19. Incoming and outgoing leads are connected to the effective dimer and the steady-state transport coefficient of the bosons is inferred from the evaluation of the scattered wave function of the leads due to the presence of the dimer.

The paper is organized as follows. In Sec. 2] we present the physical system of specific interest and the model that we adopt to evaluate on-site and hopping energies of the 1D Bose-Hubbard Hamiltonian. In Sec. 3 we introduce a scattering matrix formalism for the case of out-of-equilibrium leads, with the aim of calculating the bosonic transmittivity through the system. The particle current is obtained from an extrapolation using a Landauer-like formula. The method is applied for two choices of the number of fermions, in cases when they are either regularly or randomly distributed inside the lattice. The numerical results and their physical interpretation are discussed in Sec. [4 and the main conclusions are given in Sec. [5 Two Appendices give technical details on the evaluation of some parameters entering the effective 1D Hamiltonian 
and on the recursive algorithm for the calculation of the scattering matrix, while a third Appendix discusses how period doubling for a pure Bose condensate can be achieved in a four-beam experimental set-up.

\section{The model}

We focus on a mixture of ${ }^{87} \mathrm{Rb}$ and ${ }^{40} \mathrm{~K}$ atoms, which has the peculiarity of having a large and negative boson-fermion scattering length. Let us consider the case in which the number $N_{f}$ of (spin-polarized) fermions is much less than the number $N_{b}$ of bosons and at most equal to the number $n_{s}$ of available lattice sites. With a suitable choice of the laser frequency $\omega_{L}$, the lattice potential can become considerably deeper for the ${ }^{40} \mathrm{~K}$ atoms than for the ${ }^{87} \mathrm{Rb}$ atoms, so that the fermions can be taken as localized inside the lattice potential wells.

The lattice potential for the two atomic species can be written as

$$
U_{\mathrm{K}, \mathrm{Rb}}(z)=U_{\mathrm{K}, \mathrm{Rb}}^{0} \sin ^{2}(k z) \simeq-\frac{\hbar \Omega_{\mathrm{K}, \mathrm{Rb}}^{2}}{4 \delta_{\mathrm{K}, \mathrm{Rb}}} \sin ^{2}(k z)
$$

where $\delta_{\mathrm{K}, \mathrm{Rb}}=\omega_{L}-\omega_{\mathrm{K}, \mathrm{Rb}}$ is the detuning of the laser from the two atomic frequencies $\omega_{\mathrm{K}}$ and $\omega_{\mathrm{Rb}}, k=2 \pi / \lambda$ is the wave number of the laser light and $\Omega_{\mathrm{K}, \mathrm{Rb}}=d_{\mathrm{K}, \mathrm{Rb}} E_{0} / \hbar$ is the Rabi frequency, which is determined by the laser electric field $E_{0}$ and by the atomic dipole $d_{\mathrm{K}, \mathrm{Rb}}$. The period of the optical lattice is $\lambda / 2$. The atomic dipole depends on the natural width $\Gamma_{\mathrm{K}, \mathrm{Rb}}$ of the transition [20] so that the ratio $U_{\mathrm{K}}^{0} / U_{\mathrm{Rb}}^{0}$ is given by

$$
\frac{U_{\mathrm{K}}^{0}}{U_{\mathrm{Rb}}^{0}}=\frac{\Gamma_{\mathrm{K}}}{\Gamma_{\mathrm{Rb}}} \frac{\delta_{\mathrm{Rb}}}{\delta_{\mathrm{K}}}
$$

For the D2 lines of ${ }^{40} \mathrm{~K}$ and ${ }^{87} \mathrm{Rb}$, which are used in current experiments [21, 22], the natural widths are almost equal $\left(\Gamma_{\mathrm{K}} \simeq \Gamma_{\mathrm{Rb}} \simeq 6 \mathrm{MHz}\right)$, while the atomic frequencies have values $\omega_{\mathrm{K}}=390.83 \mathrm{THz}$ and $\omega_{\mathrm{Rb}}=384.227 \mathrm{THz}$. Choosing for example a laser wavelength $\lambda=763 \mathrm{~nm}$ (or $\omega_{L}=392.88 \mathrm{THz}$ ) the ratio between the depths of the two lattice potentials is $U_{\mathrm{K}}^{0} / U_{\mathrm{Rb}}^{0}=4.26$, so that the transport of the fermions can be neglected in a regime where tunnelling motions of the bosons are still allowed.

However, the presence of the localized fermions affects the transmittivity of the bosons by modifying the effective potential that the latter see. In the case of a negative mutual scattering length a well containing a fermion becomes deeper for the bosons inside it, so that these bosons will have a lower probability of hopping to the next well.

To study the effect of the fermions on the bosonic transport as indicated above, we build a 1D tight-binding Hamiltonian for the bosons and use a Green's function approach to evaluate their transport coefficient through the lattice. The Bose-Hubbard Hamiltonian for the bosons is

$$
H_{I}=\sum_{i=1}^{n_{s}}\left[E_{i}|i\rangle\langle i|+\gamma_{i}(|i\rangle\langle i+1|+| i+1\rangle\langle i|)\right] .
$$

Here, the parameters $E_{i}$ and $\gamma_{i}$ depend on the number of bosons in the lattice well labelled by the index $i$ and represent site energies and hopping energies, respectively.

We proceed to a 1D reduction of the Hamiltonian by introducing the transverse widths $\sigma_{\perp \mathrm{Rb}, \mathrm{K}}$ of the bosonic and fermionic wave functions in a cigar-shaped harmonic trap, together with a $1 \mathrm{D}$ condensate wave function $\phi_{i}(z)$ in the $i-t h$ cell and the 
1D fermion density $n_{f}(z)$. In a tight-binding scheme $\phi_{i}(z)$ is a Wannier function for the bosons in the potential $U_{\mathrm{Rb}}(z)$ given in Eq. (11) and, according to the early work of Slater [23], can be written as a Gaussian function,

$$
\phi_{i}(z)=\phi_{i}(0) \exp \left[-\left(z-z_{i}\right)^{2} /\left(2 \sigma_{z \mathrm{Rb}}^{2}\right)\right]
$$

where $\left|\phi_{i}(0)\right|^{2}$ is the number of bosons in the lattice well $i$. The fermion density is similarly written as a superposition of Gaussian functions localized in a set of lattice wells labelled by an index $i^{\prime}$,

$$
n_{f}(z) \propto \sum_{i^{\prime}} \exp \left[\left(z-z_{i^{\prime}}\right)^{2} / \sigma_{z \mathrm{~K}}^{2}\right]
$$

The determination of the widths $\sigma_{\perp \mathrm{Rb}, \mathrm{K}}$ and $\sigma_{z \mathrm{Rb}, \mathrm{K}}$ is carried out variationally, as described in Appendix A

We can now evaluate the parameters entering the effective Hamiltonian. The site energies are given by

$$
E_{i}=\int d z \phi_{i}^{2}(z)\left[-\frac{\hbar^{2} \nabla^{2}}{2 m_{\mathrm{Rb}}}+U_{\mathrm{Rb}}(z)+\frac{1}{2} g_{b b}\left|\phi_{i}(z)\right|^{2}+g_{b f} n_{f}(z)-m_{\mathrm{Rb}} a z+C_{\mathrm{Rb}}\right]
$$

where $m_{\mathrm{Rb}}$ is the ${ }^{87} \mathrm{Rb}$ mass, $a=F / m_{\mathrm{Rb}}$ is the acceleration due to a constant external force $F$ acting on the bosons, $C_{\mathrm{Rb}}=\hbar^{2} /\left(2 m_{\mathrm{Rb}} \sigma_{\perp \mathrm{Rb}}^{2}\right)+\frac{1}{2} m_{\mathrm{Rb}} \omega_{\perp \mathrm{Rb}}^{2} \sigma_{\perp \mathrm{Rb}}^{2}$, and $\omega_{\perp \mathrm{Rb}}$ is the radial frequency of the harmonic trapping potential. The parameters $g_{b b}$ and $g_{b f}$ are the strengths of the $1 \mathrm{D}$ boson-boson and boson-fermion interactions, which are given by

$$
\left\{\begin{aligned}
g_{b b} & =\frac{4 \pi \hbar^{2}}{m_{\mathrm{Rb}}} \frac{a_{b b}}{2 \pi \sigma_{\perp \mathrm{Rb}}^{2}} \\
g_{b f} & =\frac{2 \pi \hbar^{2}}{m_{r}} \frac{a_{b f}}{\pi\left(\sigma_{\perp \mathrm{Rb}}^{2}+\sigma_{\perp \mathrm{K}}^{2}\right)}
\end{aligned}\right.
$$

with $a_{b b}, a_{b f}$ the boson-boson and boson-fermion scattering lengths 2 and $m_{r}$ the boson-fermion reduced mass. Consistently with the model, the hopping energies $\gamma_{i}$ are given by

$$
\gamma_{i}=\int d z \phi_{i}(z)\left[-\frac{\hbar^{2} \nabla^{2}}{2 m_{\mathrm{Rb}}}+U_{\mathrm{Rb}}(z)+\frac{1}{2} g_{b b}\left|\phi_{i}(z)\right|^{2}+g_{b f} n_{f}(z)+C_{\mathrm{Rb}}\right] \phi_{i+1}(z) .
$$

This completes the determination of the effective 1D Hamiltonian for the bosons, that we shall use to evaluate the transmittivity for various distributions of the fermions in the lattice wells.

\section{The transmittivity}

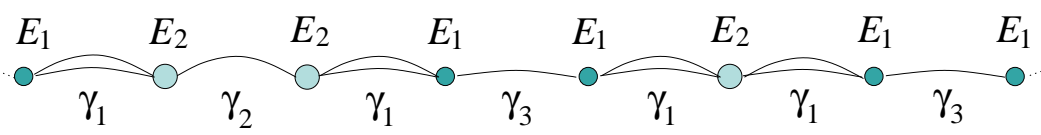

Figure 1. Schematic representation of the tight-binding Hamiltonian for a condensate in a 1D lattice, with some sites containing a fermion.

In Fig. 10 shew a schematic representation of the Hamiltonian (3) in the absence of an external force. For such a system the boson site energies can be of two types, $E_{1}$ 
and $E_{2}$ depending on the absence or presence of a fermion in the well, and the boson hopping energies can take three values: $\gamma_{1}$ if the hopping is between a site with a fermion and a site without, $\gamma_{2}$ in the case of two neighboring wells which both contain a fermion, and $\gamma_{3}$ in the case of two neighboring wells without fermions. We shall consider the following situations: (i) the fermionic band is empty (EF), namely no fermions are present in the lattice; $(i i)$ the fermionic band is filled (FF), namely there is one fermion per well; (iii) the fermionic band is half-filled $\left(N_{f}=n_{s} / 2\right)$ with every other well occupied in an orderly way (OHF); and $(i v)$ the fermionic band is half-filled but the fermions are distributed on the lattice in a disordered way (DHF).

In the tight-binding regime the system of bosons behaves in the first two cases as a one-band system, which is described by a single site energy $\left(E_{1}\right.$ in the first case and $E_{2}$ in the second) and by a single hopping energy ( $\gamma_{3}$ and $\gamma_{2}$, respectively). The third case describes a two-band boson system with two alternating site energies $\left(E_{1}\right.$ and $\left.E_{2}\right)$ and a single hopping energy $\left(\gamma_{1}\right)$. There is in this case a folding of the dispersion relation in quasi-momentum space, with the opening of an energy minigap $\Delta E=\left|E_{2}-E_{1}\right|$ between two sub-bands of equal energy width. In the fourth case the bosons again form a two-band system of the type illustrated in Fig. [1 1 and the presence of disorder will cause a broadening of the two bands. We show in Appendix B how the third case could be realized in the laboratory by using a four-beam set-up instead of an ordered array of fermionic atoms at half occupancy. The forth case could be realized by adding some speckle to the lattice [16.
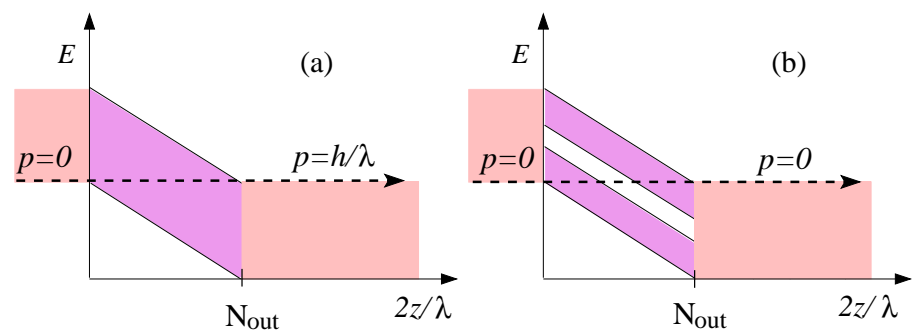

Figure 2. Band tilting due to a constant force $F$ and coupling of the condensate to an incoming and an outgoing lead for the case of (a) a one-band system and (b) a two-band system. The position $z$ is in units of $\lambda / 2$.

The effect of applying a constant force to the condensate can be described as a tilting of the bands in coordinate space, due to the induced position dependence of the site energies. This is shown in Fig. 22 the situation is analogous to that obtained in an electron gas on a lattice by applying to it a static, uniform electric field [24]. The transmittivity of such a system can be calculated, following the proposal of Farchioni et al. [25, through an evaluation of the scattering matrix which explicitly takes into account the presence of the bias [26. For this purpose, let us connect the system to incoming and outgoing leads which mimic its coupling to the continuum by injecting and extracting a steady-state particle current, respectively. The bosons are initially in their lowest momentum state $(p=0)$ and can leave the lattice only at $p=h / \lambda$ (in the one-band case) or at $p=0$ (in a small-minigap two-band system) if the coupling to the leads is effected as is shown in Fig. 22 Elastic Bragg scattering can also occur at both these points in quasi-momentum space as well as at the top of the lower subband in the split-band configuration. As we shall see, in the latter case this produces resonances in the transmittivity. 
Let us define $N_{\text {out }}$ (with $N_{\text {out }} \leq n_{s}$ ) as the number of hops after which the condensate reaches the highest energy point in the dispersion curve and can either leave the lattice or be Bragg reflected. The two leads have a band width $4 \gamma_{L}$ and their centers at $E_{L}^{\text {in }}$ and $E_{L}^{\text {out }}$ are shifted by $F N_{\text {out }} \lambda / 2$ in order to optimize the coupling with the lattice. The outgoing wavefunction $\phi_{\text {out }}^{\tilde{\kappa}}(z)$ is an eigenstate of the outgoing lead and is connected to the incoming wavefunction $\phi_{\mathrm{in}}^{\kappa}(z)$, which is an eigenstate of the incoming lead, by the relation

$$
\phi_{\text {out }}^{\tilde{\kappa}}(z)=\Omega^{+}(E) \phi_{\text {in }}^{\kappa}(z) \text {. }
$$

The wavevectors $\kappa$ and $\tilde{\kappa}$ of the two wavefunctions are uniquely identified by the relation

$$
\kappa, \tilde{\kappa}=\frac{2}{\lambda} \arccos \left(\frac{E-E_{L}^{\mathrm{in}, \text { out }}}{2 \gamma_{L}}\right) .
$$

The operator $\Omega^{+}(E)=\mathbb{I}+G^{0}(E) T(E)$ in Eq. (9) involves the Green's function $G^{0}(E)=\left(E-H_{0}\right)^{-1}$ of the two leads, as well as the scattering matrix $T(E)=$ $H_{I}\left[\mathbb{I}-G^{0}(E) H_{I}\right]^{-1}$ where $H_{I}$ is the Hamiltonian acting on the first $N_{\text {out }}$ sites of the lattice subject to the force $F$ (see Appendix C).

In the next step we renormalize the interaction Hamiltonian $H_{I}$ into an effective Hamiltonian $\tilde{H}_{I}(E)$ acting in the subspace $\left\{|1\rangle,\left|N_{\text {out }}\right\rangle\right\}$ formed by the two edge sites that are directly connected to the leads. In this renormalization approach the scattering matrix is also projected on the subspace $\left\{|1\rangle,\left|N_{\text {out }}\right\rangle\right\}$ and can be written as a $2 \times 2$ matrix and evaluated with a recursive algorithm as described in Appendix C. In such a formalism the transmittivity coefficient $\mathcal{T}(E)$, which is defined as

$$
\mathcal{T}(E)=\frac{\lim _{n \rightarrow+\infty}\left\langle n \mid \phi_{\text {out }}^{\tilde{\kappa}}\right\rangle\left\langle\phi_{\text {out }}^{\tilde{\kappa}} \mid n\right\rangle v_{\text {out }}}{\lim _{m \rightarrow-\infty}\left\langle m \mid \phi_{\text {in }}^{\kappa}\right\rangle\left\langle\phi_{\text {in }}^{\kappa} \mid m\right\rangle v_{\text {in }}}
$$

with $v_{\text {in }}$ and $v_{\text {out }}$ the velocities of the incoming and outgoing wavefunction, is related to the off-diagonal element $T(E)_{1, N_{\text {out }}}$ of the scattering matrix by

$$
\mathcal{T}(E)=4 \frac{\left|T(E)_{1, N_{\text {out }}}\right|^{2}}{\gamma_{L}^{2}} \sin \left(\kappa \frac{\lambda}{2}\right) \sin \left(\tilde{\kappa} \frac{\lambda}{2}\right) .
$$

It is easy to show that Eq. (12) is equivalent to the trace of Eq. (63) in the work of Paulsson [26].

The transmittivity determines the particle current passing through the lattice, and to calculate it we can exploit Landauer's approach adapted to a bosonic gas with out-of-equilibrium leads. Again following Paulsson [26], the current $j$ can be written

$$
j=\frac{N_{b}}{\pi \hbar n_{s}} \int \mathrm{d} E\left[f\left(E-\mu_{1}\right)-f\left(E-\mu_{2}\right)\right] \mathcal{T}(E)
$$

where $f(E-\mu)$ is the Bose-Einstein distribution and $\mu_{1,2}$ are the chemical potentials of the reservoirs which act as the source and the sink of the bosons. Note that the normalization $N_{b} / n_{s}$ of the bosonic wavefunction plays the role of the electric charge in the Landauer formula [27. In the zero-temperature limit we have

$$
f\left(E-\mu_{1}\right)-f\left(E-\mu_{2}\right) \approx \frac{\partial f(E-\mu)}{\partial E}\left(\mu_{2}-\mu_{1}\right)
$$

and the density probability $\partial f(E-\mu) / \partial E$ is a delta function $\delta(E-\mu)$. Then Eq. (13) gives

$$
j=\frac{N_{b}}{\pi \hbar n_{s}} \mathcal{T}(\bar{\mu}) \Delta \mu
$$


where $\Delta \mu=\mu_{2}-\mu_{1}=N_{\text {out }} F \lambda / 2$ is the difference in chemical potentials and $\bar{\mu}=\left(\mu_{1}+\mu_{2}\right) / 2$ is their average value, corresponding to the dashed line in Fig. 2

The transmittivity may also be expressed in terms of the number of bosons exiting from the lattice as a drop emitted under a constant driving force, as in the experiment of Anderson and Kasevich [5]. The current is written in that context as

$$
j=N_{\mathrm{drop}} \nu_{B},
$$

where $\nu_{B}=(F \lambda) /(2 h)$ is the frequency of Bloch oscillations and $N_{\text {drop }}$ is the number of bosons in the first drop. This can be calculated from Eqs. (1516) as

$$
\frac{N_{\text {drop }}}{N_{b}}=\frac{N_{\text {out }}}{n_{s}} \mathcal{T}(\bar{\mu}) \text {. }
$$

Equation (17) holds for a steady-state current and will not be applicable to the subsequent drops unless the condensate is continuously replenished.

\section{Results}

We have calculated the transmittivity of the model as a function of the acceleration $a$ for the four cases listed at the beginning of Sec. 3] The difference $\Delta \mu$ of the chemical potentials of the two leads does not depend on $a$ and is fixed by the total band width $4 \gamma_{L}$ of the leads, so that the current $j$ is directly proportional to the transmittivity (see Eq. (15)). We have chosen to focus on the typical experimental parameters used at LENS [21] for the ${ }^{87} \mathrm{Rb}^{40} \mathrm{~K}$ mixture, setting $N_{b}=10^{5}, n_{s}=200, U_{\mathrm{Rb}}^{0}=3.5 E r_{\mathrm{Rb}}$, and $\omega_{\perp \mathrm{Rb}}=2 \pi \times 85.7 \mathrm{~Hz}$. As previously remarked, we set $\lambda=763 \mathrm{~nm}$ so that we can consider each ${ }^{40} \mathrm{~K}$ atom as localized in a lattice well. Moreover, for this choice of the laser wavelength, the detunings $\delta_{\mathrm{K}, \mathrm{Rb}}$ are much larger than the natural widths $\Gamma_{\mathrm{K}, \mathrm{Rb}}$ and spontaneous emission can be neglected.

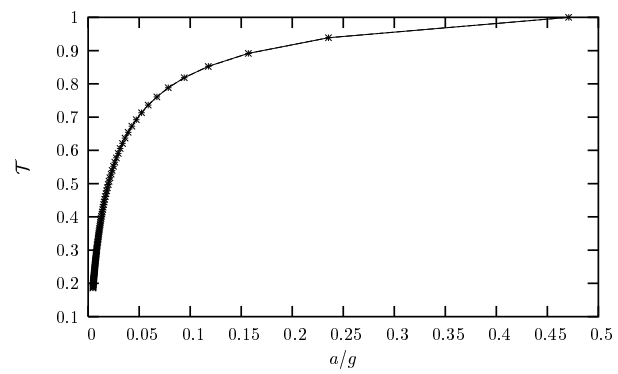

Figure 3. The transmittivity as a function of the acceleration $a$ (in units of the acceleration of gravity $g$ ) for the cases of an empty fermion band (EF: + ) and a completely filled fermion band (FF: $\mathrm{X})$. The lines are guides to the eyes.

In Fig. 3 we show the transmittivity of a one-band system as a function of $a$ for the one-band cases in which either the fermions are absent (EF) or each well is occupied by one fermion (FF). The effect of the presence of the fermions should be that the boson-fermion attractions diminish the hopping probability of the bosons between two neighboring wells, so that the transmittivity should be lowered. However, for the case of only one fermion per well and for the usual values of the boson-fermion scattering length, this effect is not visible (see Fig. 31). 

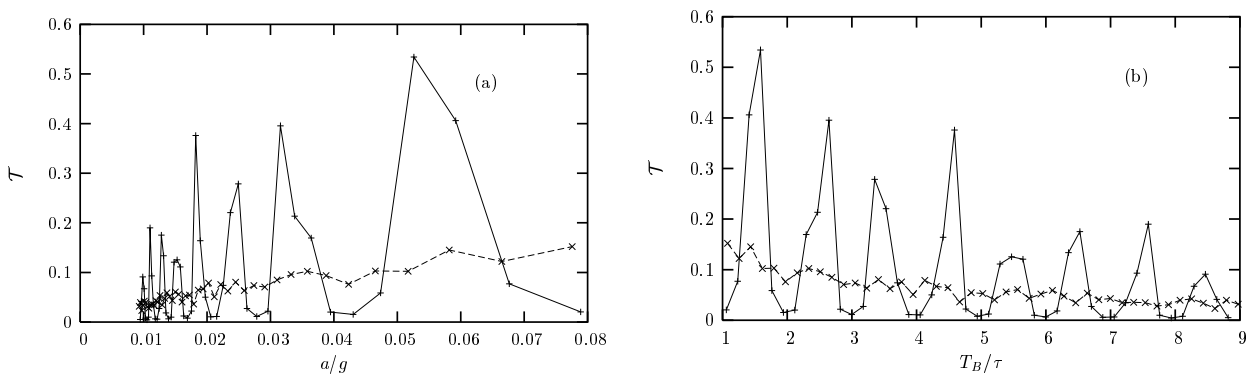

Figure 4. (a) The transmittivity at half occupancy as a function of the acceleration $a$ (in units of the acceleration of gravity $g$ ) for an ideal two-band system (OHF: +) and for randomly distributed fermions (DHF: X). (b) The same transmittivity data are plotted against the ratio $T_{B} / \tau$. The lines are guides to the eyes.

In Fig. 4 4 we show the transmittivity of the two-band system for $N_{f}=100$ fermions which are distributed in the lattice wells either regularly (OHF) or completely at random (DHF). The value of $N_{\text {out }}$ in these calculations has been taken to run from

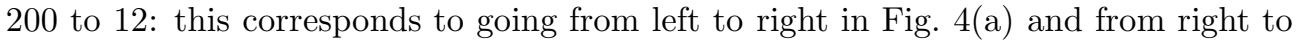
left in Fig. 目(b). The transmittivity in Fig. 目 is on average strongly reduced relative to the cases shown in Fig. [3] but in the OHF case it shows a very prominent structure of peaks and troughs. The observed pattern arises from the interference occuring at $p=-h / 2 \lambda$ (the left-hand edge of the Brillouin zone in the split-band configuration) between the boson wave packets that are Bragg scattered at $p=h / 2 \lambda$ in the lower sub-band and those that have tunnelled into the upper sub-band, travelled through it, and tunnelled back into the lower sub-band at $p=-h / 2 \lambda$. The two times that govern the interference pattern are the Bloch oscillation period $T_{B}=h /(F \lambda)$ and the time $\tau$ taken by the bosons in coherently tunnelling twice across the minigap, which is proportional to $\left(\hbar N_{b} / \Delta E n_{s}\right)$ [28. We expect constructive interference in the reflectivity at $p=0$ in the upper sub-band, i.e. destructive interference in the transmittivity, whenever the ratio $T_{B} / \tau$ is an integer number. This behaviour is illustrated in Fig. 目(b) by replotting the transmittivity data against $T_{B} / \tau$ with the choice $\tau=\left(3 \pi^{2} / 8\right)\left(\hbar N_{b} / \Delta E n_{s}\right)$. Of course this specific value of the proportionality constant in the tunnelling time, which is consistent with the Heisenberg principle, depends on the model Hamiltonian that we have assumed.

Returning to Fig. 目(a), the effect of disorder in the distribution of the fermions in the half-occupancy DHF case is to strongly diminish the contrast in the interference

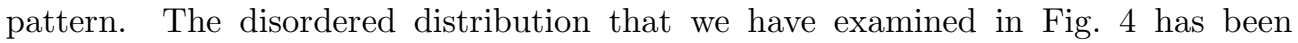
produced from a random number generator and the pattern is completely washed out in this case of maximum disorder. While the average occupancy of a site by a fermion is only one-half, the transmittivity of the bosons is greatly reduced relative to the FF case in Fig. 3. Evidently, the main effect at play is due to the fluctuations in the disordered distribution of the fermions, which tend to break the coherence of the Bose condensate. 


\section{Concluding remarks}

In conclusion, we have studied the condensate current induced by a constant external force in a 1D optical lattice in the presence of various numbers of fermionic atoms, distributed over the lattice in orderly or random ways. We have focused on the ${ }^{87} \mathrm{Rb}-$ ${ }^{40} \mathrm{~K}$ mixture, with a choice of the laser generating the lattice potential such that the barriers for the fermions are much higher than those for the Bose condensate. In this regime fermionic transport can be neglected, but the presence of the fermions and their spatial distribution affect the bosonic transport via mean-field attractive interactions.

We have calculated the transmittivity and the condensate current without explicitly diagonalizing the Bose-Hubbard Hamiltonian, but by exploiting an out-ofequilibrium Green's function approach adapted to the bosonic case. When there is one fermion per well, the current is essentially the same as in the absence of fermions and increases monotonically with the driving force. When every second well is occupied by a fermion, instead, the energy spectrum breaks into two sub-bands and the probability that the condensate may tunnel through the band minigap depends on the energy that it has acquired at the edge of the first sub-band and on the energy width of the minigap. Part of the condensate is back-scattered when it arrives at the edge of the lower sub-band and can interfere at the opposite edge of the Brillouin zone with the part that has tunnelled to the upper sub-band and travelled through it. This gives rise to an interference pattern showing marked maxima and minima in the transmittivity, whose location is an integer multiple of a parameter directly proportional to the energy minigap and inversely proportional to the driving force.

The interference pattern loses resolution when the distribution of the fermions in the lattice becomes disordered, up to being completely washed out in the case of full randomness. An ordered period doubling of the lattice could be realized, however, with a four-beam experimental set-up. Observation of resonances in the current as a function of the driving force would allow a measurement of the tunnelling time through the minigap.

\section{Acknowledgments}

This work was supported by INFM through the PRA2001-Photonmatter. ZA also acknowledges support from TUBITAK and from the Research Fund of the University of Istanbul under Project Number BYP-110/12122002.

\section{Appendix A. Determination of radial and axial widths}

We adopt the variational method proposed for Bose condensates by Salasnich et al. 18. Within a 1D nonpolynomial nonlinear Schrödinger equation approach, we find that the evaluation of the transverse widths $\sigma_{\perp \mathrm{Rb}, \mathrm{K}}$ requires the self-consistent solution of the Euler-Lagrange equations

$$
\left\{\begin{array}{l}
\frac{\hbar^{2}}{2 m_{\mathrm{Rb}} \sigma_{\perp \mathrm{Rb}}^{2}}-\frac{1}{2} m_{\mathrm{Rb}} \omega_{\perp \mathrm{Rb}}^{2} \sigma_{\perp \mathrm{Rb}}^{2}+\frac{1}{2} g_{b b}\left|\phi_{i}(z)\right|^{2}+g_{b f} \frac{\sigma_{\perp \mathrm{Rb}}^{2}}{\sigma_{\perp \mathrm{Rb}}^{2}+\sigma_{\perp \mathrm{K}}^{2}} n_{f}(z)=0 \\
\frac{\hbar^{2}}{2 m_{\mathrm{K}} \sigma_{\perp \mathrm{K}}^{2}}-\frac{1}{2} m_{\mathrm{K}} \omega_{\perp \mathrm{K}}^{2} \sigma_{\perp \mathrm{K}}^{2}+g_{b f} \frac{\sigma_{\perp \mathrm{K}}^{2}}{\sigma_{\perp \mathrm{Rb}}^{2}+\sigma_{\perp \mathrm{K}}^{2}}\left|\phi_{i}(z)\right|^{2}=0 .
\end{array}\right.
$$

Assuming that $\sigma_{\perp \mathrm{Rb}, \mathrm{K}}$ are essentially constant along the axial position, we set in Eqs. A.1 $\left|\phi_{i}(z)\right|^{2} \simeq N_{b} /\left(\sqrt{\pi} n_{s} \sigma_{z \mathrm{Rb}}\right)$ and $n_{f}(z) \simeq 1 /\left(\sqrt{\pi} \sigma_{z \mathrm{~K}}\right)$ or $n_{f}(z)=0$, 
depending on the presence or absence of a fermion in the corresponding well. The axial widths $\sigma_{z \mathrm{Rb}, \mathrm{K}}$ entering the axial densities depend themselves on the radial widths $\sigma_{\perp \mathrm{Rb}, \mathrm{K}}$. Consequently we first solve Eqs. (A.1) for given values of $\sigma_{z \mathrm{Rb}, \mathrm{K}}$, evaluated in the absence of interactions and then, having calculated the radial widths, we evaluate the correction to the axial widths.

Minimization of the bosonic and fermionic energies with respect to $\sigma_{z} \mathrm{Rb}, \mathrm{K}$ yields the two coupled equations

$$
\left\{\begin{array}{l}
\frac{\hbar^{2}}{2 m_{\mathrm{Rb}} \sigma_{z \mathrm{Rb}}^{2}}-\frac{1}{2} m_{\mathrm{Rb}} \omega_{z \mathrm{Rb}}^{2} \sigma_{z \mathrm{Rb}}^{2}+\frac{\hbar^{2}}{2 m_{\mathrm{Rb}} \sigma_{z \mathrm{Rb}}^{2}}-\frac{g_{b b} N_{b}}{2 \sqrt{2 \pi} n_{s} \sigma_{z \mathrm{Rb}}}+\frac{g_{b f}}{\sqrt{\pi}} \frac{\sigma_{z \mathrm{Rb}}^{2}}{\left(\sigma_{z \mathrm{Rb}}^{2}+\sigma_{z \mathrm{~K}}^{2}\right)^{3 / 2}}=0 \\
\frac{\hbar^{2}}{2 m_{\mathrm{K}} \sigma_{z \mathrm{~K}}^{2}}-1_{2} m_{\mathrm{K}} \omega_{z \mathrm{~K}}^{2} \sigma_{z \mathrm{~K}}^{2}+\frac{g_{b f} N_{b}}{\sqrt{\pi} n_{s}} \frac{\sigma_{z \mathrm{~K}}^{2}}{\left(\sigma_{z \mathrm{Rb}}^{2}+\sigma_{z \mathrm{~K}}^{2}\right)^{3 / 2}}=0
\end{array}\right.
$$

for the case of a well containing a fermion, while in the absence of a fermion we have

$$
\frac{\hbar^{2}}{2 m_{\mathrm{Rb}} \sigma_{z \mathrm{Rb}}^{2}}-\frac{1}{2} m_{\mathrm{Rb}} \omega_{z \mathrm{Rb}}^{2} \sigma_{z \mathrm{Rb}}^{2}+\frac{g_{b b} N_{b}}{2 \sqrt{2 \pi} n_{s} \sigma_{z \mathrm{Rb}}}=0
$$

as already found by Chiofalo et al. 13. Here, the axial frequencies $\omega_{z \mathrm{Rb}, \mathrm{K}}$ for a single well are expressed in terms of the potential depths $U_{\mathrm{Rb}, \mathrm{K}}^{0}$ and of the recoil energies $E r_{\mathrm{Rb}, \mathrm{K}}=\hbar^{2} k^{2} / 2 m_{\mathrm{Rb}, \mathrm{K}}$ as

$$
\omega_{z \mathrm{Rb}, \mathrm{K}}=\frac{1}{\hbar} \sqrt{4 U_{\mathrm{Rb}, \mathrm{K}}^{0} E r_{\mathrm{Rb}, \mathrm{K}}} .
$$

The solution of Eqs. (A.1)- (A.3) provides the further input that is needed in Eqs. (6) and (8).

\section{Appendix B. Period doubling with a four-beam set-up}

Four laser beams can be used to create a 1D lattice with a double periodicity along the $z$ direction. Two beams are set in a counterpropagating configuration along the $z$ axis, while the other two beams are rotated by angles of $60^{\circ}$ and $120^{\circ}$ with respect to the $z$ axis. In the region of space where the four beams are superposed, the longitudinal electric field $E$ is proportional to

$$
E \propto \beta e^{i \alpha}\left(e^{i k z / 2}+e^{i \phi_{1}} e^{-i k z / 2}\right)+\left(e^{i k z}+e^{i \phi_{2}} e^{-i k z}\right) .
$$

In Eq. (B.1) $\beta$ and $\alpha$ are the relative amplitude and phase of the rotated beams with respect to the counterpropagating pair, $\phi_{1}$ is the relative phase of the rotated beams, and $\phi_{2}$ the relative phase of the counterpropagating beams. All these parameters can be set by choosing suitable optical components.

Upon setting $\phi_{1}=\phi_{2}=\pi$ and $\alpha=\pi / 2$, the resulting lattice potential will have the shape

$$
U(z) \propto \beta^{2} \sin ^{2}(k z / 2)+\sin ^{2}(k z)
$$

and the double period enters through the well depths, that is the site energies, which will differ by a factor $\beta^{2}$. Instead, by setting $\phi_{1}=\phi_{2}=0$ and $\alpha=\pi / 2$ we have

$$
U(z) \propto \beta^{2} \cos ^{2}(k z / 2)+\cos ^{2}(k z)
$$

and the potential hills (that is the hopping energies) have alternating values. In the case of a ${ }^{87} \mathrm{Rb}$ condensate, a lattice potential of the type in Eq. (B.2) with $\beta \simeq 0.1$ is equivalent to the effective potential generated by a regular distribution of ${ }^{40} \mathrm{~K}$ atoms inside every other well of an optical lattice created with a single pair of laser beams. 


\section{Appendix C. The recursive algorithm}

Figure C1 shows a schematic representation of the whole Hamiltonian for the system composed of a finite lattice and two leads, and of its decomposition into the sum of $H_{0}$ and $H_{I}$ for the evaluation of the scattering matrix. We show below how $H_{I}$ can be reduced to an effective Hamiltonian $\tilde{H}_{I}$ for a dimer in a subspace $\left\{|1\rangle,\left|N_{\text {out }}\right\rangle\right\}$ and how this leads to an expressions for the scattering matrix and for the outgoing wavefunctions.

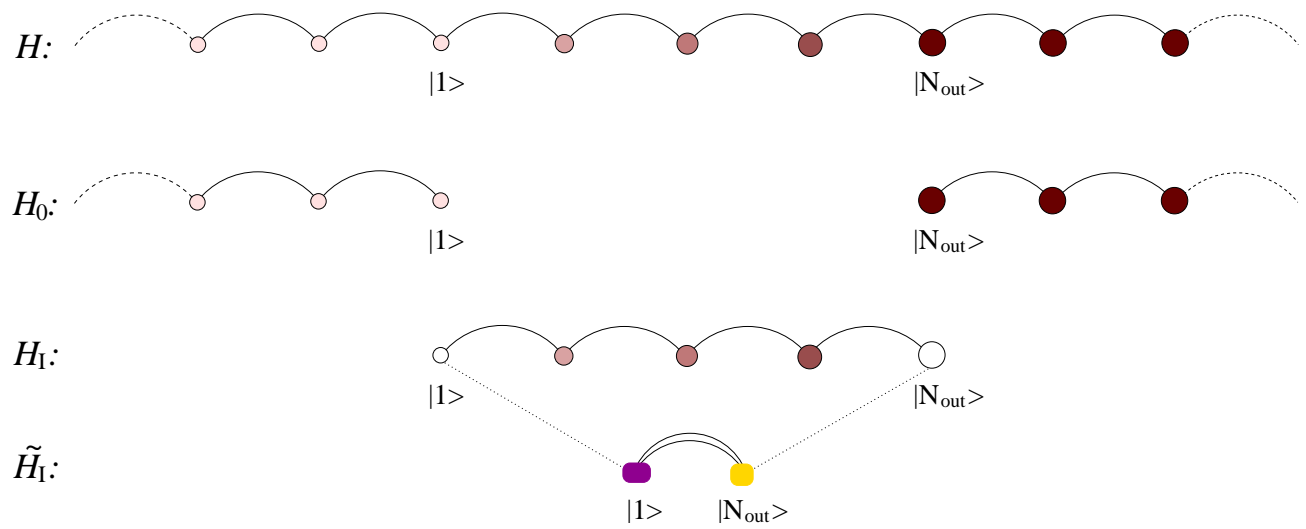

Figure C1. Schematic representation of the Hamiltonian $H=H_{0}+H_{I}$ of the system composed by the leads $\left(H_{0}\right)$ and by the lattice $\left(H_{I}\right)$, and of the effective Hamiltonian $\tilde{H}_{I}$ projected on the two edge sites.

Let $n$ be the site index number. To calculate the transmitted wavefunction we have to evaluate

$$
\left\langle n \mid \phi_{\text {out }}^{\tilde{\kappa}}\right\rangle=\sum_{m}\left\langle n\left|\mathbb{I}+G^{0}(E) T(E)\right| m\right\rangle\left\langle m \mid \phi_{\text {in }}^{\kappa}\right\rangle
$$

for $n>N_{\text {out }}$. Since the wavefunctions $\phi_{\text {in }}^{\kappa}$ and $\phi_{\text {out }}^{\tilde{\kappa}}$ are defined in two disconnected spaces, Eq. (C.1) becomes

$$
\begin{aligned}
\left\langle n \mid \phi_{\text {out }}^{\tilde{\kappa}}\right\rangle & =\sum_{m \leq 1, l}\left\langle n\left|G^{0}(E)\right| l\right\rangle\langle l|T(E)| m\rangle\left\langle m \mid \phi_{\text {in }}^{\kappa}\right\rangle \\
& =\left\langle n\left|G^{0}(E)\right| N_{\text {out }}\right\rangle\left\langle N_{\text {out }}|T(E)| 1\right\rangle\left\langle 1 \mid \phi_{\text {in }}^{\kappa}\right\rangle \\
& =\sqrt{2}\left[G^{0}(E)\right]_{n, N_{\text {out }}}[T(E)]_{N_{\text {out }}, 1} \sin (\kappa \lambda / 2) u_{\kappa}(\lambda / 2),
\end{aligned}
$$

where we have set $\left\langle 1 \mid \phi_{\text {in }}^{\kappa}\right\rangle=u_{\kappa}(\lambda / 2)[\exp (i \kappa \lambda / 2)-\exp (-i \kappa \lambda / 2)] / i \sqrt{2}$ with $u_{\kappa}(\lambda / 2)=$ $\phi_{i}(z)$. It can be shown that Eq. (C.2) is equivalent to Eq. (34) in the work of Paulsson [26] by making use of the relation $G^{0}(E) T(E)=G(E) H_{I}(E)$.

The Green's function element $\left[G^{0}(E)\right]_{n, N_{\text {out }}}$ in Eq. (C.2) determines the coherence between site $n$ and site $N_{\text {out }}$ on the chain for the outgoing lead. It can be written as

$$
\left[G^{0}(E)\right]_{n, N_{\text {out }}}=\frac{\gamma_{L}^{n-N_{\text {out }}}}{\mid \gamma_{L}^{n+1-N_{\text {out }} \mid}} e^{i \tilde{\kappa}\left(n+1-N_{\text {out }}\right) \lambda / 2}
$$


in terms of quantities defined in the main text. The scattering matrix element $[T(E)]_{N_{\text {out }}, 1}$ can be evaluated from the effective Hamiltonian $\tilde{H}_{I}(E)$ as schematically illustrated in Fig. [C1]

$$
[T(E)]_{N_{\text {out }}, 1}=\left\langle N_{\text {out }}\left|\tilde{H}_{I}(E)\left[\mathbb{I}_{2}-\tilde{G}^{0}(E) \tilde{H}_{I}(E)\right]^{-1}\right| 1\right\rangle
$$

where $\mathbb{I}_{2}$ is the $2 \times 2$ identity matrix and $\tilde{G}^{0}(E)$ is the Green's function of the leads projected onto the subspace $\left\{|1\rangle,\left|N_{\text {out }}\right\rangle\right\}$. The only non-zero elements of the $2 \times 2$ matrix $\tilde{G}^{0}(E)$ are the diagonal ones, which are given by $\mathcal{G}\left(E-E_{L}^{\text {in }}, \gamma_{L}\right)$ and $\mathcal{G}\left(E-E_{L}^{\text {out }}, \gamma_{L}\right)$, with

$$
\mathcal{G}\left(E-E_{s}, \gamma_{L}\right)=\frac{1}{2 \gamma_{L}^{2}}\left[E-E_{s}-\sqrt{\left(E-E_{s}\right)^{2}-4 \gamma_{L}^{2}}\right] .
$$

Finally, the elements of the matrix $\tilde{H}_{I}(E)$ in Eq. (C.4) can be calculated by decimation of the sites $\left(2,3, \ldots, N_{\text {out }}-1\right)$ and can be written in the form of continued fractions. That is,

$$
\left[\tilde{H}_{I}(E)\right]_{1,1}=\left[H_{I}\right]_{1,1}+\frac{\left[H_{I}\right]_{1,2}^{2}}{E-\left[H_{I}\right]_{2,2}-\frac{\left[H_{I}\right]_{2,3}^{2}}{E-\left[H_{I}\right]_{3,3}-\ldots}}
$$

and

$$
\left[\tilde{H}_{I}(E)\right]_{1, N_{\text {out }}}=\left[H_{I}\right]_{1,2} \frac{1}{E-\left[H_{I}\right]_{2,2}}\left[H_{I}\right]_{2,3} \frac{1}{E-\left[H_{I}\right]_{3,3}-\frac{\left[H_{I}\right]_{3,2}^{2}}{E-\left[H_{I}\right]_{2,2}}}\left[H_{I}\right]_{3,4} \ldots,(\mathrm{C} .7
$$

with $\left[\tilde{H}_{I}(E)\right]_{N_{\text {out }}, 1}=\left[\tilde{H}_{I}(E)\right]_{1, N_{\text {out }}}$ and $\left[\tilde{H}_{I}(E)\right]_{N_{\text {out }}, N_{\text {out }}}$ given similarly to $\left[\tilde{H}_{I}(E)\right]_{1,1}$ in Eq. (C.6).

\section{References}

[1] Mølmer K 1998 Phys. Rev. Lett. 80 1804; Akdeniz Z, Vignolo P, Minguzzi A and Tosi M P 2002 J. Phys. B 35 L105

[2] Modugno G, Roati G, Riboli F, Ferlaino F, Brecha R J, and Inguscio M 2002 Science 2972240

[3] Ferlaino F, Brecha R J, Hannaford P, Riboli F, Roati G, Modugno G, and Inguscio M, cond-mat/0211051

[4] Ben Dahan M, Peik E, Reichel J, Castin Y, and Salomon C 1996 Phys. Rev. Lett. 76 4508; Peik E, Ben Dahan M, Bouchoule I, Castin Y, and Salomon C 1997 Phys. Rev. A 552989

[5] Anderson B P and Kasevich M A 1998 Science 2821686

[6] Bharucha C F, Madison K W, Morrow P R, Wilkinson S R, Sundaram B and Raizen M G 1997 Phys. Rev. A 55 R857

[7] Orzel C, Tuchman A K, Fenselau M L, Yasuda M, and Kasevich M A 2001 Science 2912386

[8] Cataliotti F S, Burger S, Fort C, Maddaloni P, Minardi F, Trombettoni A, Smerzi A, and Inguscio M 2001 Science 293843

[9] Greiner M, Mandel O, Esslinger T, Hänsch T W, and Bloch I 2002 Nature 41539

[10] Steinhauer J, Katz N, Ozeri R, Davidson N, Tozzo C, and Dalfovo F cond-mat/0209075

[11] Chiofalo M L, Succi S, and Tosi M P 1999 Phys. Lett. A 260 86; Chiofalo M L and Tosi M P 2000 Phys. Lett. A 268 406; Cardenas M, Chiofalo M L, and Tosi M P 2002 Physica B 322 116; Adhikari S K and Muruganandam P cond-mat/0209429

[12] Smerzi A, Trombettoni A, Kevrekidis P G, and Bishop A R 2002 Phys. Rev. Lett. 89170402

[13] Berg-Sørensen K and Mølmer K 1998 Phys. Rev. A 58 1480; Chiofalo M L, Polini M, and Tosi M P 2000 Eur. Phys. J. D 11, 371; van Oosten D, van der Straten P, and Stoof H T C 2001 Phys. Rev. A 63053601 
[14] Jaksch D, Bruder C, Cirac J I, Gardiner C W, and Zoller P 1998 Phys. Rev. Lett. 813108

[15] Prokof'ev N V, Svistunov B V, and Tupitsyn I S 1998 Phys. Lett. A 238 253; cond-mat/9901083

[16] Damski B, Zakrzewski J, Santos L, Zoller P, and Lewenstein M cond-mat/0303065

[17] Potting S, Cramer M, Schwalb C H, Pu H, and Meystre P 2001 Phys. Rev. A 64023604

[18] Salasnich L, Parola A, and Reatto L 2002 Phys. Rev. A 65043614

[19] Grosso G and Pastori Parravicini G 1986 Adv. Chem. Phys. 62 81; ibid. 62 131; Giannozzi P, Grosso G, Moroni S, and Pastori Parravicini G 1988 Appl. Numer. Math. 4273

[20] Atom-Photon Interactions: Basic Processes and Applications, edited by Cohen-Tannoudji C, Dupont-Roc J, and Grynberg G (Wiley-Interscience, New York 1998)

[21] Roati G, Riboli F, Modugno G, and Inguscio M 2002 Phys. Rev. Lett. 89 150403; Fort C, Cataliotti F S, Fallani L, Ferlaino F, Maddaloni P, and Inguscio M cond-mat/0210240

[22] Goldwin J, Papp S B, deMarco B, and Jin D S 2002 Phys. Rev. A 65021402

[23] Slater J C 1952 Phys. Rev. 87807

[24] Zener C 1934 Proc. R. Soc. London A 145523

[25] Farchioni R, Vignolo P, and Grosso G 1999 Phys. Rev. B 6015705

[26] Paulsson M cond-mat/0210519

[27] Landauer R 1970 Philos. Mag. 21863

[28] Sakurai J J, Modern Quantum Mechanics (Addison Wesley, Reading 1994) 